\section{Prävention bei Asthma bronchiale im Kindesalter}

\section{Kabesch, E. von Mutius}

Kinderklinik und Poliklinik im Dr. von Haunerschen Kinderspital Innenstadt-Klinikum der Universität München

\section{Prevention of asthma in childhood}

Environmental factors during early childhood seem to influence the onset of asthma, a disease that affects $10 \%$ of all German children, respectively. Prevention strategies are needed to reduce the burden of the disease. Breast feeding and the avoidance of environmental tobacco smoke exposure are recommended for primary prevention. Furthermore, secondary prevention measurements are suggested to reduce the onset of symptoms, once the disease is present.
Pulmonale Erkrankungen stellen bei Kindern in Deutschland den häufigsten Grund für Arztbesuche dar. Asthma bronchiale, mit einer Prävalenz von $10 \%$ eine der häufigsten chronischen Erkrankungen im Kindesalter in Deutschland, nimmt dabei eine entscheidende Rolle ein. Studien der letzten Jahre haben zu- dem gezeigt, dass die Prävalenz der Erkrankung weltweit im Anstieg begriffen ist. Hochrechnungen basierend auf Zahlen des Jahres 1992 beziffern die aufgrund von Asthma bronchiale entstehenden jährlichen Kosten (direkt und indirekt) für das Gesundheitssystem mit 5,13 Milliarden DM (Pneumologie 1996;50:
Tab. 1 Zusammenfassung der Risikofaktoren für die Entstehung und Exazerbation von Asthma bronchiale.

\begin{tabular}{|c|c|c|}
\hline $\begin{array}{l}\text { Genetische } \\
\text { Prädispo- } \\
\text { sition }\end{array}$ & $\begin{array}{l}\text { gesicherter Risikofak } \\
\text { Der Anteil der geneti } \\
\text { Es handelt sich um eir } \\
\text { Genen }\end{array}$ & $\begin{array}{l}\text { r für die Entstehung von Asthma und Allergie. } \\
\text { ken Prädisposition an der Krankheitsentstehung wird auf bis zu 75\% geschätzt. } \\
\text { komplexes, polygenes Vererbungsmuster mit der Beteiligung von zahlreichen }\end{array}$ \\
\hline \multirow[t]{10}{*}{$\begin{array}{l}\text { Umwelt- } \\
\text { faktoren }\end{array}$} & Passivrauchen & $\begin{array}{l}\text { gesicherter Risikofaktor für die Entstehung von Asthma und die } \\
\text { Exazerbation von Symptomen }\end{array}$ \\
\hline & Ernährung & $\begin{array}{l}\text { ungesicherte Hinweise, dass Ernährungsfaktoren wie ungesättigte } \\
\text { Fettsäuren (Fischkonsum), Antioxidantien (Vitamin C) NaCl und Magnesium } \\
\text { die Entstehung von Asthma beeinflussen könnten. }\end{array}$ \\
\hline & Stillen & $\begin{array}{l}\text { protektiver Einfluss von Stillen auf die Allergie-Entstehung und möglicherweise } \\
\text { auf die Entstehung von Asthma sehr wahrscheinlich }\end{array}$ \\
\hline & $\begin{array}{l}\text { Luftver- } \\
\text { schmutzung }\end{array}$ & $\begin{array}{l}\text { wichtiger Risikofaktor für die Exazerbation von Asthmasymptomen, } \\
\text { kein gesicherter Risikofaktor für die Krankheitsentstehung }\end{array}$ \\
\hline & Ozon & wichtiger Risikofaktor für die Exazerbation von Asthmasymptomen \\
\hline & Sozialstatus & $\begin{array}{l}\text { Surrogat für Lifestyle und Lebensbedingungen: unterschiedliche } \\
\text { Assoziationen in verschiedenen Populationen }\end{array}$ \\
\hline & Familiengröße & $\begin{array}{l}\text { Hinweise für eine Risikoreduktion für die Entstehung von Asthma bronchiale } \\
\text { mit der Zunahme der Geschwisterzahl }\end{array}$ \\
\hline & Allergenexposition & wichtiger Risikofaktor für die Exazerbation von Asthmasymptomen \\
\hline & $\begin{array}{l}\text { Frühe mikrobielle } \\
\text { Exposition }\end{array}$ & $\begin{array}{l}\text { potentiell protektiver Faktor für die Entstehung von Allergie und } \\
\text { Asthma bronchiale }\end{array}$ \\
\hline & RSV Infektion & $\begin{array}{l}\text { Prädiktor für die Entstehung von Asthma bronchiale, kausaler } \\
\text { Zusammenhang nicht bestätigt }\end{array}$ \\
\hline
\end{tabular}

364-71). Anstrengungen, durch Prävention zu einer Eindämmung der Krankheit beizutragen, sind daher nicht nur im Interesse der Patienten zu sehen, sondern haben auch Auswirkungen gesundheitsökonomischer Natur.

Asthma wird als komplexe, multifaktorielle Erkrankung angesehen, bei der die folgenden wesentlichen Charakteristika vorhanden sind: Entzündung der Atemwege, bronchiale Hyperreaktivität gegenüber endogenen oder exogenen Stimuli und eine reversible bronchiale Obstruktion. Dabei besteht in den meisten Fällen eine signifikante Assoziation mit atopischen Phänomenen, wie der Erhöhung von Immunglobulin E. Die Entstehung der Erkrankung wird sowohl durch die individuelle genetische Veranlagung als auch durch Umweltfaktoren beeinflusst (Monatsschr Kinderheilkd 2001; 149:94-105). Als genetische Prädisposition wird dabei die Gesamtheit der komplexen Veränderungen in all jenen Genen angesehen, die an pathophysiologischen Mechanismen zur Krankheitsentstehung beteiligt sind. Eine Reihe von Umwelteinflüssen, die Risikofaktoren für Asthma bronchiale und Allergie darstellen, wurden in epidemiologischen Studien identifiziert (J Allergy Clin Immunol. 2000; 105 : 9-19). Ob es sich bei diesen Risikofaktoren um kausale Krankheitsursachen oder etwa nur um Surrogate für andere Einflussfaktoren handelt, ist in vielen Fällen noch ungeklärt (Tab.1). So ist zum Beispiel eine hohe Belastung mit Hausstaubmilben gewiss ein potenter Auslöser von Asthmasymptomen, jedoch ist der ursächliche Zusammenhang mit der Krankheitsentstehung von Asthma und Allergie nicht gesichert.

\section{Primäre Prävention}

Es erscheint sinnvoll, alle Möglichkeiten der Prävention durch Beeinflussung von krankheitsrelevanten Umweltfaktoren zu nutzen. Da davon ausgegangen werden muss, dass die Weichen für die Entstehung von Asthma bronchiale bereits sehr früh gestellt werden (Thorax 2001; 56: 153-157), muss auch jeder Versuch der primären Prävention (im Sinne von 
Verhinderung der Krankheitsentstehung) sehr früh ansetzten.

\section{Vermeidung von Passivrauchexposition}

Ein Weg der primären Prävention, der Erfolg verspricht, ist die frühe Reduktion der Passivrauchexposition von Kindern. Dies beginnt bereits mit der Forderung nach einer rauchfreien Umgebung während der fetalen Entwicklung und setzt sich auch postpartal fort. Aufklärung der Mütter, dass Rauchen während der Schwangerschaft ein eindeutiger Risikofaktor für eine verringerte Lungenfunktion und für ein erhöhtes Asthmarisiko des Kindes darstellt, steht dabei im Vordergrund. Das Aufwachsen in einem rauchfreien Zuhause sollte zu den Grundrechten jedes Kindes zählen. Diese Expositionsprophylaxe ist bei Kindern, die eine familiäre Vorbelastung hinsichtlich Asthma aufweisen, umso wichtiger (Thorax 1999; 54: 357-366).

\section{Allergenkarenz}

Als weitere primäre Präventionsmaßnahme wurde versucht, die Allergenexposition bereits pränatal zu verringern, um so die Entstehung von Asthma bronchiale zu reduzieren (Lancet 2001; 358:188-193). Die Ergebnisse der bisher dazu vorliegenden Studien lassen jedoch noch keinen eindeutig positiven Effekt dieser Maßnahmen erkennen. Ebenso scheint die frühe postpartale Allergenreduzierung nicht in der Lage, die Entstehung von Asthma bronchiale eindeutig und längerfristig anhaltend zu verringern (Lancet 2000; 356: 1392-1397).

\section{Stillen und Ernährung}

Eine Meta-Analyse von 12 prospektiven Studien aus den Jahren 1966 bis 1999 zeigte einen eindeutig protektiven Effekt von Stillen auf die Entstehung von Asthma in der Kindheit (J Pediatr 2001; 139: 261-266). Dieser Effekt war bei Kindern mit einer familiären Vorbelastung durch Asthma am deutlichsten ausgeprägt und reduzierte nach dieser Analyse das Asthmarisiko um die Hälfte. Es kann also davon ausgegangen werden, dass Stillen einen zumindest vorübergehend protektiven Effekt auf die Asthmaentstehung hat.

Als Alternative zum Stillen stehen hypoallergene Säuglingsnahrungen (Hydrolysate) zur Verfügung, die das Risiko für die Entstehung von Asthma und Allergie bei Kindern mit einer positiven Familienanamnese verringern sollen. Die dazu vorliegenden Ergebnisse beschränken sich jedoch nur auf den relativ kurzen Zeitraum der ersten Lebensjahre und lassen so noch keine Beurteilung der Auswirkung von Hydrolysaten auf die spätere Asthmaentstehung zu (GINI Studie). So wurde gezeigt, dass die frühe Sensibilisierung gegen Nahrungsmittelallergene und die damit assoziierte frühkindliche Neurodermitis durch Hydrolysaternährung reduziert werden konnte. Daten zur Auswirkungen auf die spätere Asthmaprävalenz werden in den nächsten Jahren von Kohortenstudien erwartet.

\section{Sekundäre Prävention}

Im Gegensatz zur primären ist die sekundäre Prävention von Asthma unumstritten und sollte Bestandteil jeder Asthma-

Tab. 2 Wirksamkeit von präventiven Maßnahmen in der primären und sekundären Prävention von Asthma bronchiale.

\begin{tabular}{lll}
\hline Maßnahmen & primär & sekundär \\
\hline Allergenreduktion & & \\
pränatal & nicht gesichert & \\
postnatal & nicht gesichert & sehr gut wirksam \\
Stillen & möglicherweise wirksam & \\
Hydrolysate & möglicherweise wirksam & \\
Lactobacillus & nicht gesichert & \\
Reduktion von Rauch/Passivrauchexposition & wirksam & sehr gut wirksam \\
\hline
\end{tabular}

behandlung sein. Die sekundäre Prävention soll bei bereits bestehender Erkrankung und Diagnosestellung das Auftreten von neuerlichen Symptomen reduzieren und eine dauerhafte Schädigung der Lunge verhindern. Im Grunde kann auch die Dauerbehandlung mit entzündungshemmenden Medikamenten als sekundäre Prophylaxe angesehen werden, die im Zentrum der Asthmatherapie stehen sollte. Darüber hinaus hat sich eine Reihe von Präventionsmaßnahmen als durchaus geeignet erwiesen, das Ziel einer Symptomreduzierung zu erreichen. Dazu zählt auch die Vermeidung von Passivrauchexposition, die neben der primären Präventionswirkung auch dazu beiträgt, bei bereits bestehendem Asthma das Auftreten von Symptomen zu verringern.

\section{Reduktion der Allergenexposition}

Während der Stellenwert der Allergenreduktion in der primären Prävention noch unklar ist, gibt es an der Effektivität dieser Maßnahme zur Verhinderung von Asthmasymptomen keine Zweifel. Liegt eine klinisch relevante Sensibilisierung gegen ein bestimmtes Allergen vor, so sind geeignete Methoden zur Allergenvermeidung nötig (Br Med Bull 2000; 56 : 1071-1086). Im Falle der Sensibilisierung gegen Hausstaubmilbenallergene ist vor allem die Reduktion der Milbenexposition im Schlafbereich wichtig. Dazu gehören in erster Linie milbendichte Kopfkissen und Matratzenbezüge ebenso wie die Milbenreduktion bei Stofftieren im Kinderbett. Besteht die Möglichkeit, so sollten Teppichböden aus dem Schlafzimmer entfernt werden. Ebenso tragen Staubsauger mit speziellen Allergenfiltern zur Reduktion der Allergenbelastung beitragen. Liegt eine symptomatische Allergie gegen Haustiere vor, sollten diese am 
Info

Ansprechpartner

Gesellschaft für pädiatrische Pneumologie

e.V.

Vorsitzender: Prof. Dr. med. D. Reinhardt

Kinderklinik und Kinderpoliklinik

im Dr. von Haunerschen Kinderspital

Lindwurmstraße $2 \mathrm{a}$

80337 München

Tel.: 089/5160-7700

Fax: 089/5160-7702

E-Mail: dietrich.reinhard@

kk-i.med.uni-muenchen.de

\section{DGAI}

Deutsche Gesellschaft für Allergologie und Klinische Immunologie e.V.

Präsident: Prof. Dr. med. Johannes Ring

Klinikund Poliklinikfür Dermatologie und

Allergologie

der Technischen Universität München

Biedersteiner Straße 29

80802 München

Tel.: 089/4140-3170

Fax: 089/4140-3171

Email: Johannes.Ring@Irz.tum.de

Ansprechpartner:

RatzingerErika@t-online.de

Deutsche Gesellschaft für Pneumologie

(DGP)

c/o Medizinische Klinik und Poliklinik

BG-Kliniken

Bergmannsheil-Universitätsklinik

Bürkle-de-la-Camp-Platz 1

44789 Bochum

E-Mail: gerhard.schulze-werninghaus@

ruhr-uni-bochum.de

Mittelpunkt dieser Immunreaktionslage, die vor allem zur Abwehr von bakteriellen und viralen Erregern dient) nötig. Erste Studien, die dieses Ziel verfolgen, liegen bereits vor. Dabei wurde an atopische Mütter während der Schwangerschaft Lactobacillus GG verabreicht, um so das Immunsystem des Feten bereits pränatal Richtung TH-1 Immunantwort zu stimulieren (Lancet 2001; 357: 1076-1079). Während dabei die Daten zur Reduzierung von atopischem Ekzem im ersten Lebensjahr vielversprechend $\mathrm{zu}$ sein scheinen, lässt sich die Auswirkung dieser präventiven Behandlung auf die Entstehung von Asthma noch nicht beurteilen. Vor einer Anwendung dieser Präventionsstrategie auf breiter Basis sind daher sicherlich noch weitere Untersuchungen abzuwarten, die vor allem auch die longitudinalen Auswirkungen dieser Maßnahmen berücksichtigen müssen.

\section{Fazit}

Es kann davon ausgegangen werden, dass Maßnahmen der sekundären Prävention eine gesicherte Wirkung besitzen und daher als notwendiger Teil der Asthma-Therapie empfohlen werden (Tab. 2). Mit Ausnahme der Passivrauchreduktion ist die Wirksamkeit von primären Präventionsmaßnahmen nicht gesichert. Daher können die Maßnahmen zur primären Prävention bisher nur als Präventionsversuche gewertet werden.

Korrespondenz:

Dr. Michael Kabesch

Dr. von Haunersches Kinderspital

Lindwurmstraße 4

80337 München

Tel.: 089/5160 2792

Fax: 089/5160 4764

Michael.Kabesch@

kk-i.med.uni-muenchen.de

\section{Fachliche Betreuung der \\ "Serie Prävention“:}

Prof. Dr. Dr. h. c. Peter C. Scriba

Medizinische Klinik / Klinikum Innenstadt

der Universität München

Ziemssenstr. 1

80336 München

Prof. Dr. Friedrich W. Schwartz

Medizinische Hochschule Hannover

Abteilung für Epidemiologie, Sozialmedizin

und Gesundheitsforschung

Carl-Neuberg-Str. 1

30623 Hannover rung einer TH-1 dominierten Immunantwort (T-Helferzellen des Typs 1 stehen im 